\title{
A Novel Cat Swarm Optimization Algorithm for Unconstrained Optimization Problems
}

\author{
Meysam Orouskhani \\ Department of Computer Engineering, Science and Research Branch, Islamic Azad University, Tehran Iran \\ E-mail: orouskhani@ce.sharif.edu
}

Yasin Orouskhani

BSc Student, Computer School of Sharif University of Technology, Tehran, Iran

E-mail: orouskhani@gmail.com

Mohammad Mansouri

Intelligent System Laboratory (ISLAB), Electrical and Computer engineering department, K.N.Toosi University E-mail: mohammad_mansouri@ee.kntu.ac.ir

Mohammad Teshnehlab

Industrial Control Center of Excellence, Faculty of Electrical and Computer Engineering, K.N. Toosi University E-mail: teshnehlab@ee.kntu.ac.ir

\begin{abstract}
Cat Swarm Optimization (CSO) is one of the new swarm intelligence algorithms for finding the best global solution. Because of complexity, sometimes the pure CSO takes a long time to converge and cannot achieve the accurate solution. For solving this problem and improving the convergence accuracy level, we propose a new improved CSO namely 'Adaptive Dynamic Cat Swarm Optimization'. First, we add a new adaptive inertia weight to velocity equation and then use an adaptive acceleration coefficient. Second, by using the information of two previous/next dimensions and applying a new factor, we reach to a new position update equation composing the average of position and velocity information. Experimental results for six test functions show that in comparison with the pure CSO, the proposed CSO can takes a less time to converge and can find the best solution in less iteration.
\end{abstract}

Index Terms - Swarm Intelligence, Cat Swarm Optimization, Evolutionary Algorithms

\section{Introduction}

Function Optimization is one of the important fields in the computational intelligence theories. There are many algorithms to find the global and local solutions of the problems. So me of these optimization algorith ms were developed based on swarm intelligence. These algorithms imitate the creature's swarm behavior and model into algorithm, such as Ant Colony Optimization (ACO) which imitates the behavior of ants ${ }^{[1]-[6]}$, Particle Swarm Optimization (PSO) which imitates the behavior of birds ${ }^{[2]}$, Bee Colony Optimization (BCO) which imitates the behavior of bees ${ }^{[3]}$ and the recent finding, Cat Swarm Optimization (CSO) which imitates the behavior of cats ${ }^{[4]}$

By simulating the behavior of cats and modeling into two modes, CSO can solve the optimization problems. In the cases of functions optimization, CSO is one of the best algorithms to find the global solution. In comparis on with other heuristic algorithms such as PSO and PSO with weighting factor ${ }^{[7]}$, CSO usually achieves better result. But, because of algorithm complexity, solving the problems and finding the optimal solution may take a long process time and sometimes much iteration is needed.

So in this article, we propose an improved CSO in order to achieve the high convergence accuracy in less iteration. First we use an adaptive inertia weight and adaptive acceleration coefficient. So, the new velocity update equation will be computed in an adaptive formula. Then, our aim is to consider the effect of previous/next steps in order to calculate the current position. So by using a factor namely 'Forgetting Factor', information values of steps will be different. Finally, we use an average form of position update equation composing new velocity and position information. Experimental results for standard optimization benchmarks indicate that the proposed algorithm rather than pure CSO can improve performance on finding the best global solution and achieves better accuracy level of convergence in less iteration. 
The rest of article is organized as follows: in section 2 basic of CSO is reviewed in two phases. Section 3 introduces new CSO with adaptive parameters and novel position equation. Experimental results and comparison of proposed CSO with pure are discussed in last section.

\section{Cat Swarm Optimization}

Cat Swarm Optimization is a new optimization algorith $m$ in the field of swarm intelligence ${ }^{[4]}$. The CSO algorithm models the behavior of cats into two modes: 'Seeking mode' and 'Tracing mode'. Swarm is made of initial population composed of particles to search in the solution space. For example, we can simu late birds, ants and bees and create Particle swarm optimization, Ant colony optimization and Bee colony optimization respectively. Here, in CSO, we use cats as particles for solving the problems.

In CSO, every cat has its own position composed of D dimensions, velocities for each dimension, a fitness value, which represents the accommodation of the cat to the fitness function, and a flag to identify whether the cat is in seeking mode or tracing mode. The final solution would be the best position of one of the cats. The CSO keeps the best solution until it reaches the end of the iterations ${ }^{[5]}$.

Cat Swarm Optimization algorithm has two modes in order to solve the problems which are described below:

\subsection{Seeking Mode}

For modeling the behavior of cats in resting time and being-alert, we use the seeking mode. This mode is a time for thinking and deciding about next move. This mode has four main parameters which are mentioned as follow: seeking memory pool (SMP), seeking range of the selected dimension (SRD), counts of dimension to change (CDC) and self-position consideration (SPC) ${ }^{[4]}$.

The process of seeking mode is describes as follow:

Step 1: Make $\mathrm{j}$ copies of the present position of cat ${ }_{\mathrm{k}}$, where $\mathrm{j}=$ SMP. If the value of SPC is true, let $\mathrm{j}=$ (SMP-1), then retain the present position as one of the candidates.

Step2: For each copy, according to CDC, randomly plus or minus SRD percent the present values and replace the old ones.

Step3: Calculate the fitness values (FS) of all candidate points.

Step4: If all FS are not exactly equal, calculate the selecting probability of each candidate point by (1), otherwise set all the selecting probability of each candidate point be 1 .

Step5: Randomly pick the point to move to from the candidate points, and replace the position of cat ${ }_{\mathrm{k}}$.

$$
\mathrm{P}_{\mathrm{i}}=\frac{\left|\mathrm{SSE}_{\mathrm{i}}-\mathrm{SSE}_{\text {max }}\right|}{\mathrm{SSE}_{\max }-\mathrm{SSE}_{\min }}
$$

If the goal of the fitness function is to find the minimum solution, $\mathrm{FS}_{\mathrm{b}}=\mathrm{FS}_{\max }$, otherwise $\mathrm{FS}_{\mathrm{b}}=\mathrm{FS}_{\text {min }}$

\subsection{Tracing Mode}

Tracing mode is the second mode of algorithm. In this mode, cats desire to trace targets and foods. The process of tracing mode can be described as follow:

Step1: Update the velocities for every dimension according to (2).

Step2: Check if the velocities are in the range of maximum velocity. In case the new velocity is overrange, it is set equal to the limit.

$$
\mathrm{V}_{\mathrm{k}, \mathrm{d}}=\mathrm{V}_{\mathrm{k}, \mathrm{d}}+\mathrm{r}_{1} \mathrm{c}_{1}\left(\mathrm{X}_{\mathrm{best}, \mathrm{d}}-\mathrm{X}_{\mathrm{k}, \mathrm{d}}\right)
$$

Step 3: Update the position of cat $\mathrm{k}_{\mathrm{k}}$ according to (3).

$$
X_{k, d}=X_{k, d}+V_{k, d}
$$

$\mathrm{X}_{\text {best, }}$ is the position of the cat, who has the best fitness value, $X_{k, d}$ is the position of cat ${ }_{k}, c_{1}$ is an acceleration coefficient for extending the velocity of the cat to move in the solution space and usually is equal to 2.05 and $r_{1}$ is a random value uniformly generated in the range of $[0,1]$.

\subsection{Core Description of CSO}

In order to co mb ine the two modes into the algorith $\mathrm{m}$, we define a mixture ratio (MR) which indicates the rate of mixing of seeking mode and tracing mode. This parameter decides how many cats will be moved into seeking mode process. For example, if the population size is 50 and the MR parameter is equal to 0.7 , there should be $50 \times 0.7=35$ cats move to seeking mode and 15 remaining cats move to tracing mode in this iteration ${ }^{[9]}$. We summarized the CSO algorithm below:

First of all, we create $\mathrm{N}$ cats and initialize the positions, velocities and the flags for cats. (*) According to the fitness function, evaluate the fitness value of the each cat and keep the best cat into memory $\left(\mathrm{X}_{\text {best }}\right)$. In next step, according to cat's flag, apply cat to the seeking mode or tracing mode process. After fin ishing the related process, re-pick the number of cats and set them into seeking mode or tracing mode according to MR parameter. At the end, check the termination condition, if satisfied, terminate the program, and otherwise go to $(*)^{[9]}$. 


\section{Modified Cat Swarm Optimization}

The tracing mode of CSO has two equations: velocity update equation and position update equation. For reaching to an adaptive $\mathrm{CSO}$, we change some of the parameters in velocity equation. Also for computing the current position of cats, we consider the information of previous and next dimensions by using a special factor and then we achieve a new dynamic position update equation. We describe the proposed algorithm in two parts.

\subsection{Using Adaptive Parameters}

In the proposed algorithm, we add an adaptive inertia weight to the velocity equation which is updated in each dimension. By using this parameter, we make a balance between global and local search ability. A large inertia weight facilitates a global search while a small inertia weigh facilitates a local search. First we use a large value and it will be reduced gradually to the least value by using (4).

$$
\mathrm{W}(\mathrm{i})=\mathrm{W}_{\mathrm{s}}+\frac{\left(\mathrm{i}_{\max }-\mathrm{i}\right)}{2 \times \mathrm{i}_{\max }}
$$

Equation (4) indicates that the inertia weight will be updated adaptively, where $\mathrm{W}_{\mathrm{s}}$ is the starting weight, $\mathrm{i}_{\max }$ is the maximum dimension of benchmark and $i$ is the current dimension. So the maximum inertia weight happens in the first dimension of the each iteration and it will be updated decreasingly in every dimension. In the proposed algorith $\mathrm{m} \mathrm{W}_{\mathrm{s}}$ is equal to 0.6 . Also, $\mathrm{c}_{1}$ is an acceleration coefficient for extending the velocity of the cat to move in the solution space. This parameter is a constant value and is usually equal to 2.05 , but we use an adaptive formula to update it by (5).

$$
C(i)=C_{s}-\frac{\left(i_{\max }-i\right)}{2 \times i_{\max }}
$$

Equation (5) demonstrates that the adaptive acceleration coefficient will be gradually increased in every dimension and the maximu $m$ value happens in the last dimension. Here $C_{s}$ is equal to 2.05. By using these two adaptive parameters, we change the velocity update equation for each cat to a new form describing in (6).

$$
\begin{aligned}
V_{k, d}=W(d) \times V_{k, d}+r_{1} & \times C(d) \\
& \times\left(X_{b e s t, d}-X_{k, d}\right)
\end{aligned}
$$

\subsection{New Dynamic Position Update Equation}

In this part, we change the position update equation to a new form. In the pure CSO, the position of cat is including of current information of velocity and position. Sometimes in many cases, using of previous information in order to estimate the current position is useful. Also, taking the advantages of next information can be appropriate information for updating the cat's position. So we use the two previous/next dimensions information of velocity and position by applying a new factor which is called 'Forgetting factor'. By this factor, the values of previous and next steps will be different. So the information value for first previous/next step is higher than second previous/next step. It means that the influence of previous/next step is more important than previous/next second step. New position update equation is described by (7).

In the proposed algorithm, $\gamma$ is the forgetting factor and is equal to 0.6 (It is necessary to use $\gamma>0.5$ ). This new position update equation is composing two new dynamic terms, average position information and average velocity information. Here, we use the current and the average information of first and second previous/next dimensions for both velocity and position by applying a forgetting factor $(\gamma)$. Figure 1 shows the process of position updating for $\mathrm{cat}_{\mathrm{k}}$.

$$
\mathrm{X}_{\mathrm{k}, \mathrm{d}}=\frac{1}{2}\left[\begin{array}{c}
(\text { Position Information }) \\
+(\text { Velocity Information })
\end{array}\right]
$$

$$
\begin{aligned}
& \text { Position Information }=\mathrm{X}_{\mathrm{k}, \mathrm{d}}+ \\
& \qquad \begin{array}{l}
\frac{\left(\gamma \times\left(\mathrm{X}_{\mathrm{k}, \mathrm{d}+1}\right)+(1-\gamma) \times\left(\mathrm{X}_{\mathrm{k}, \mathrm{d}+2}\right)\right)}{2}+ \\
\frac{\left(\gamma \times\left(\mathrm{X}_{\mathrm{k}, \mathrm{d}-1}\right)+(1-\gamma) \times\left(\mathrm{X}_{\mathrm{k}, \mathrm{d}-2}\right)\right)}{2}
\end{array}
\end{aligned}
$$

Velocity Information $=\mathrm{V}_{\mathrm{k}, \mathrm{d}}+$

$$
\begin{aligned}
& \frac{\left(\gamma \times\left(\mathrm{V}_{\mathrm{k}, \mathrm{d}+1}\right)+(1-\gamma) \times\left(\mathrm{V}_{\mathrm{k}, \mathrm{d}+2}\right)\right)}{2}+ \\
& \frac{\left(\gamma \times\left(\mathrm{V}_{\mathrm{k}, \mathrm{d}-1}\right)+(1-\gamma) \times\left(\mathrm{V}_{\mathrm{k}, \mathrm{d}-2}\right)\right)}{2}
\end{aligned}
$$




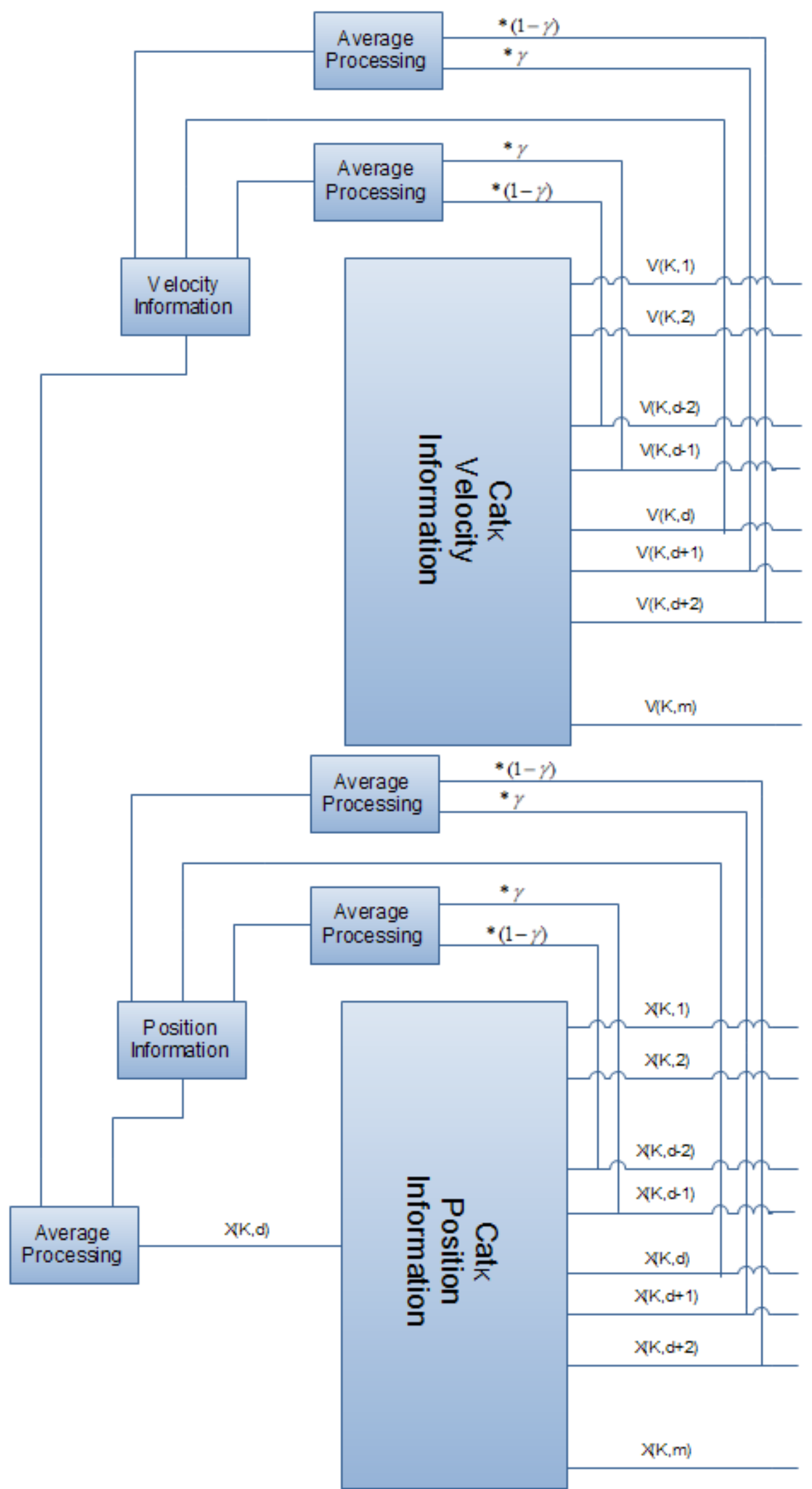

Fig. 1: Process of position updating for $\mathrm{cat}_{\mathrm{k}}$ 


\section{Experiment and Result}

In this paper, simulation experiments are conducted in GCC compiler on an Intel Core 2 Duo at $2.53 \mathrm{GHz}$ with $4 \mathrm{G}$ real memory. Our aim is to find the optimal solution of the benchmarks and find the minimum. Rastrigrin, Grie wank, Ackley, A xis parallel, Trid 10 and Zakharov are six test functions used in this work. In Table 1 and Table 2 we determine the parameter values of the pure CSO and Adaptive Dynamic CSO respectively. The limitation range of six test functions is mentioned in Table 3.
Table 1: Parameters settings for pure CSO

\begin{tabular}{|c|c|}
\hline Parameter & Range or Value \\
\hline SMP & 5 \\
\hline SRD & $20 \%$ \\
\hline CDC & $80 \%$ \\
\hline MR & $2 \%$ \\
\hline $\mathrm{C}_{1}$ & 2.05 \\
\hline $\mathrm{R}_{1}$ & {$[0,1]$} \\
\hline
\end{tabular}

Table 2: parameter settings for adaptive dynamic CSO

\begin{tabular}{|l|c|}
\hline \multicolumn{1}{|c|}{ Parameter } & Range or Value \\
\hline SMP & 5 \\
\hline SRD & $20 \%$ \\
\hline CDC & $80 \%$ \\
\hline MR & $2 \%$ \\
\hline $\mathrm{R}_{1}$ & {$[0,1]$} \\
\hline Starting Adaptive Acceleration Coefficient $\left(\mathrm{C}_{\mathrm{s}}\right)$ & 2.05 \\
\hline Starting Adaptive Inertia Weight $\left(\mathrm{W}_{\mathrm{s}}\right)$ & 0.6 \\
\hline Forgetting Factor $(\gamma)$ & 0.6 \\
\hline
\end{tabular}

Table 3: Limitation range of benchmark functions

\begin{tabular}{|l|l|c|c|}
\hline \multicolumn{1}{|c|}{ Function Name } & \multicolumn{1}{|c|}{ Limitation Range } & Minimum & Population Size \\
\hline Rastrigin & {$[2.56,5.12]$} & 0 & 160 \\
\hline Griewank & {$[300,600]$} & 0 & 160 \\
\hline Ackley & {$[-32.678,32.678]$} & 0 & 160 \\
\hline Axis Parallel & {$[-5.12,5.12]$} & 0 & 160 \\
\hline Zakharov & {$[-100,100]$} & 0 & 160 \\
\hline Trid10 & {$[-5,10]$} & -200 & 160 \\
\hline
\end{tabular}

Table 4: Experimental results of Rastrigin function

\begin{tabular}{|c|c|c|c|}
\hline Algorithm & Average Solution & Best Solution & Best Iteration \\
\hline Pure CSO & 26.64208 & 1.161127 & 2000 \\
\hline Adaptive Dynamic CSO & 18.08161 & 0 & 968 \\
\hline
\end{tabular}

Table 5: Experimental results of Griewank function

\begin{tabular}{|c|c|c|c|}
\hline Algorithm & Average Solution & Best Solution & Best Iteration \\
\hline Pure CSO & 16.34035 & 12.08197 & 2000 \\
\hline Adaptive Dynamic CSO & 6.005809 & 2.609684 & 2000 \\
\hline
\end{tabular}

Table 6: Experimental results of Ackley function

\begin{tabular}{|c|c|c|c|}
\hline Algorithm & Average Solution & Best Solution & Best Iteration \\
\hline Pure CSO & 1.031684 & 0.004589 & 3500 \\
\hline Adaptive Dynamic CSO & 1.019844 & 0 & 1264 \\
\hline
\end{tabular}


Table 7: Experimental results of Axis Parallel function

\begin{tabular}{|c|c|c|c|}
\hline Algorithm & Average Solution & Best Solution & Best Iteration \\
\hline Pure CSO & 66.38478 & 0 & 665 \\
\hline Adaptive Dynamic CSO & 49.3307 & 0 & 408 \\
\hline
\end{tabular}

Table 8: Experimental results of Zakharov function

\begin{tabular}{|c|c|c|c|}
\hline Algorithm & Average Solution & Best Solution & Best Iteration \\
\hline Pure CSO & 0.68080 & 0 & 475 \\
\hline Adaptive Dynamic CSO & 0.442817 & 0 & 275 \\
\hline
\end{tabular}

Table 9: Experimental results of Trid10 function

\begin{tabular}{|c|c|c|c|}
\hline Algorithm & Average Solution & Best Solution & Best Iteration \\
\hline Pure CSO & -125.0846 & -178.065182 & 2000 \\
\hline Adaptive Dynamic CSO & -173.384 & -194.000593 & 2000 \\
\hline
\end{tabular}

Table 10: Process time of six test functions

\begin{tabular}{|c|cc|}
\hline Algorithm & Function Name & Average Process Time \\
\hline CSO & Rastrigin & 6.4117 \\
\hline Proposed CSO & Rastrigin & 3.5074 \\
\hline CSO & Griewank & 5.6785 \\
\hline Proposed CSO & Griewank & 8.0220 \\
\hline CSO & Ackley & 8.6873 \\
\hline Proposed CSO & Ackley & 4.7585 \\
\hline CSO & Axis Parallel & 3.7814 \\
\hline Proposed CSO & Axis Parallel & 2.5203 \\
\hline CSO & Zakharov & 2.5907 \\
\hline Proposed CSO & Zakharov & 1.6085 \\
\hline CSO & Trid10 & 2.7905 \\
\hline
\end{tabular}

Process time is an important factor for us to know that whether our proposed algorithm runs as well as we expect or not. So in Table 10, we demonstrate the running time of pure $\mathrm{CSO}$ and our proposed CSO. It indicates that for six test functions, proposed CSO always runs faster than pure CSO except for Griewank function. For example, the process time of proposed $\mathrm{CSO}$ for Rastrigin function is 6.4117 which is less than pure CSO.

Rastrigin's function is based on the function of De Jong with the addition of cosine modulation in order to produce frequent local minima. Thus, the test function is highly multimodal ${ }^{[8]}$. Function has the following definition

$$
F(x)=\sum_{d=1}^{M}\left[x_{d}^{2}-10 \cos \left(2 \pi x_{d}\right)^{2}+10\right]
$$

As shown in Figure 2 and Table 4 by running the pure CSO, the best fitness value for Rastrig in function is equal to 1.161127. But the proposed algorithm can find the best optimal and the result is reached to 0 .

Griewangk function has many wide spread local minima regularly distributed ${ }^{[8]}$.Function has the following definition:

$$
F(x)=\frac{1}{4000} \sum_{d=1}^{M} x_{d}^{2}-\prod_{d=1}^{M} \cos \left(\frac{x_{d}}{\sqrt{d}}\right)+1
$$




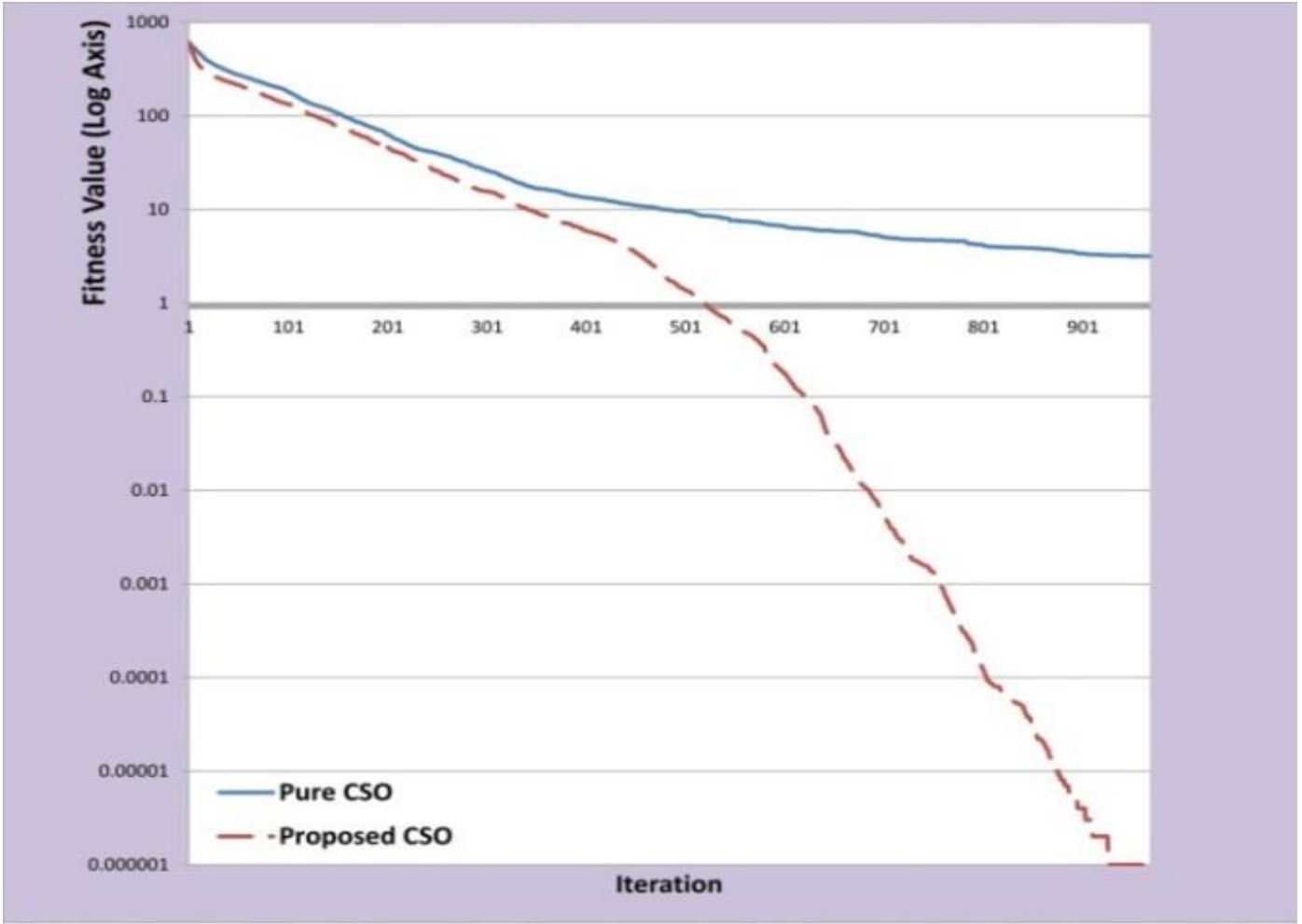

Fig. 2: Experimental Results of Rastrigin Function

As demonstrated in Figure 3 and Table 5, improved $\mathrm{CSO}$ in comparison with pure CSO, can find the better best solution in a same iteration.

Ackley's function is a widely used multimodal test function ${ }^{[8]}$. It has the following definition

$$
\begin{aligned}
\mathrm{F}(\mathrm{x})= & -20 \cdot \exp \left(-0.2 \sqrt{\frac{1}{\mathrm{n}} \sum_{\mathrm{i}=1}^{\mathrm{n}} \mathrm{x}_{\mathrm{i}}^{2}}\right) \\
& -\exp \left(\frac{1}{\mathrm{n}} \sum_{\mathrm{i}=1}^{\mathrm{n}} \cos \left(2 \pi \mathrm{x}_{\mathrm{i}}\right)\right)-20
\end{aligned}
$$

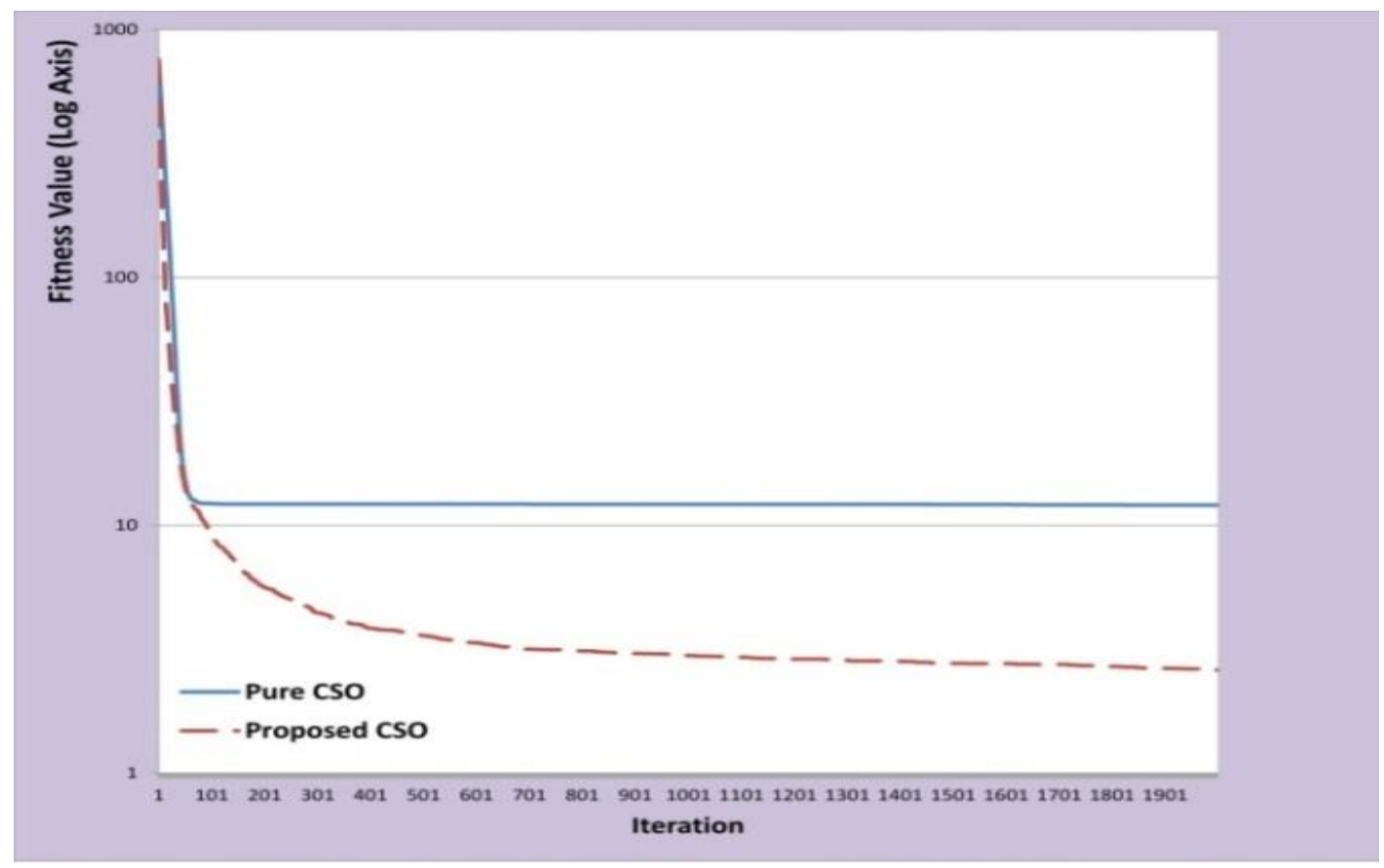

Fig. 3: Experimental Results of Griewank Function 
Figure 4 shows that the proposed CSO can find the best solution of Ackley function in less iteration. As shown, proposed CSO can get the optimal solution in $1264^{\text {th }}$ iteration. Meanwhile, the best solution of pure CSO is 0.004 in the $3500^{\text {th }}$ iteration.

The axis parallel hyper-ellipsoid is similar to function of De Jong. It is also known as the weighted sphere model. Function is continuous, convex and unimodal ${ }^{[8]}$. It has the following general definition

$$
\mathrm{F}(\mathrm{x})=\sum_{\mathrm{i}=1}^{\mathrm{n}} \mathrm{i} x_{i}{ }^{2}
$$

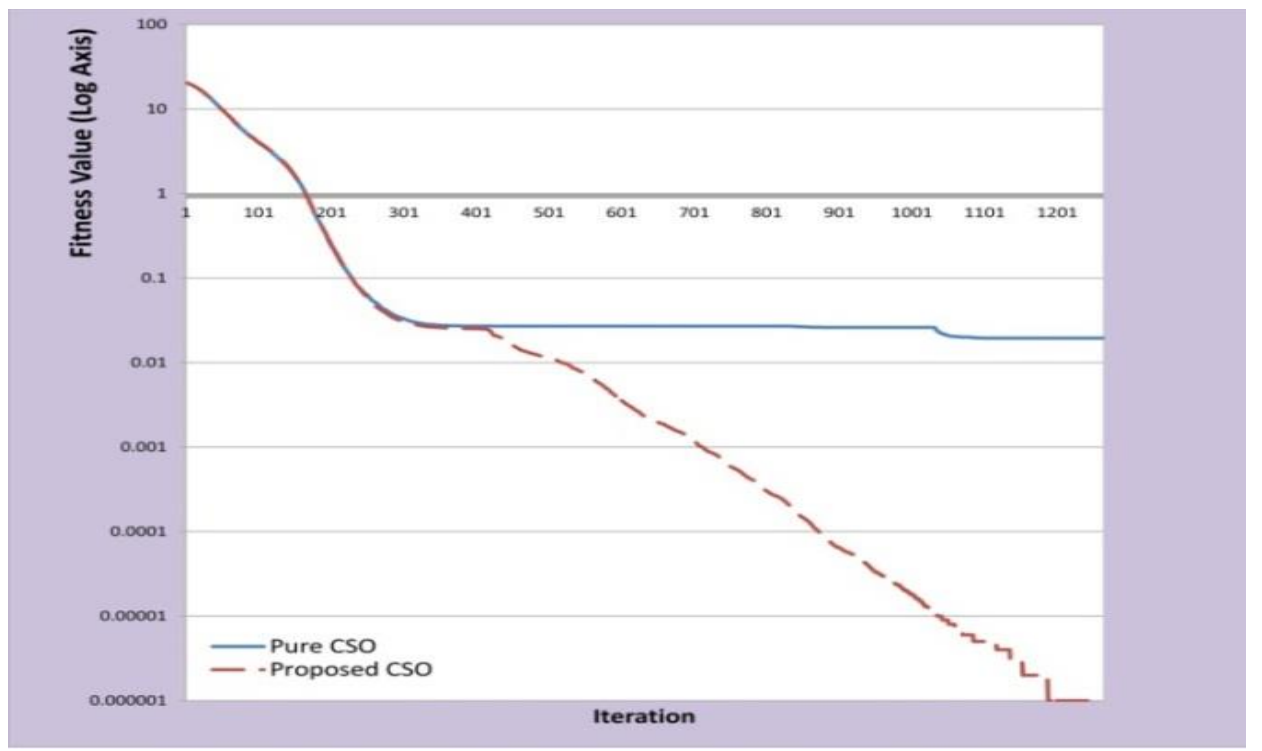

Fig. 4: Experimental Results of Ackley Function

As we shown in Figure 5 and Table 7, the optimal solution of axis parallel function is zero and both algorithms can find it. But the proposed CSO, in comparison with pure CSO can find it in less iteration.

The fifth function is Zakharov function. Zakharov equation is shown by (12).

$$
\begin{aligned}
F(x)=\sum_{i=1}^{n}\left(x_{i}\right)^{2} & +\left(\sum_{i=1}^{n} 0.5 i x_{i}\right)^{2} \\
& +\left(\sum_{i=1}^{n} 0.5 i x_{i}\right)^{4}
\end{aligned}
$$

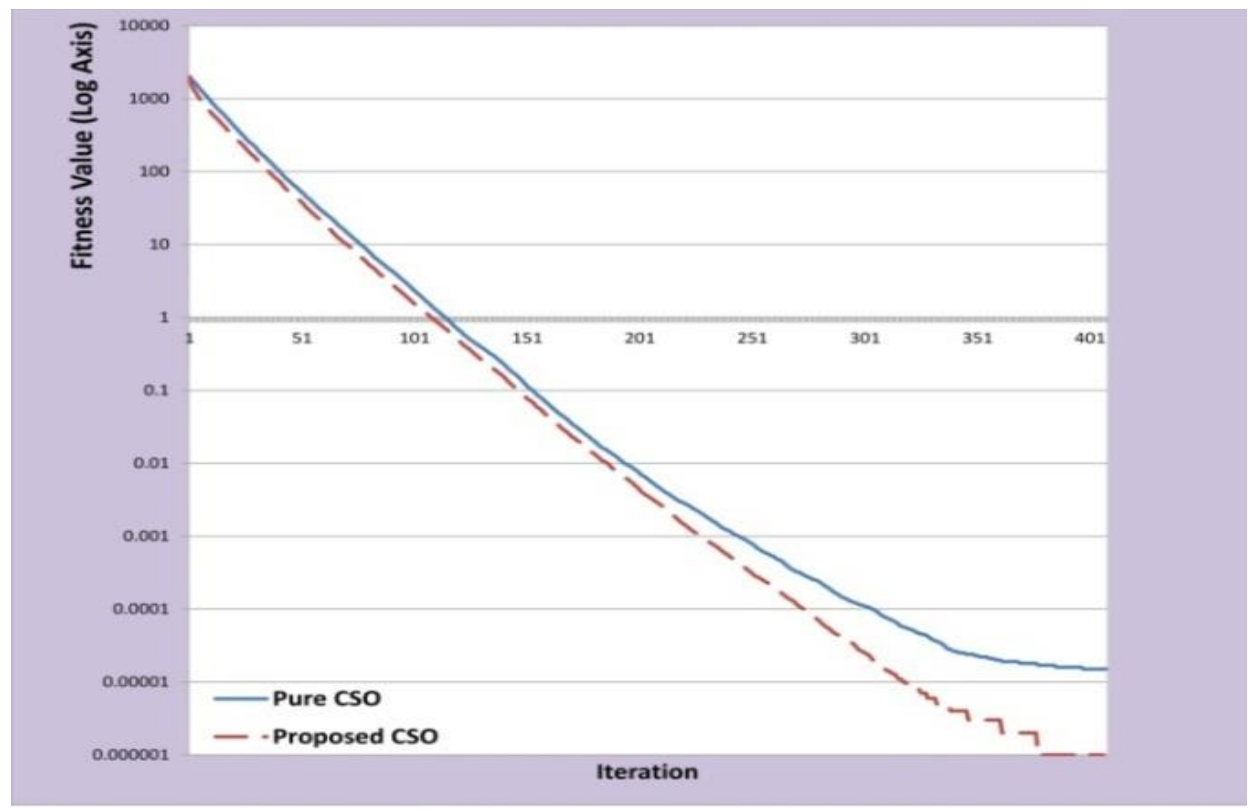

Fig. 5: Experimental Results of Axis Parallel Function 


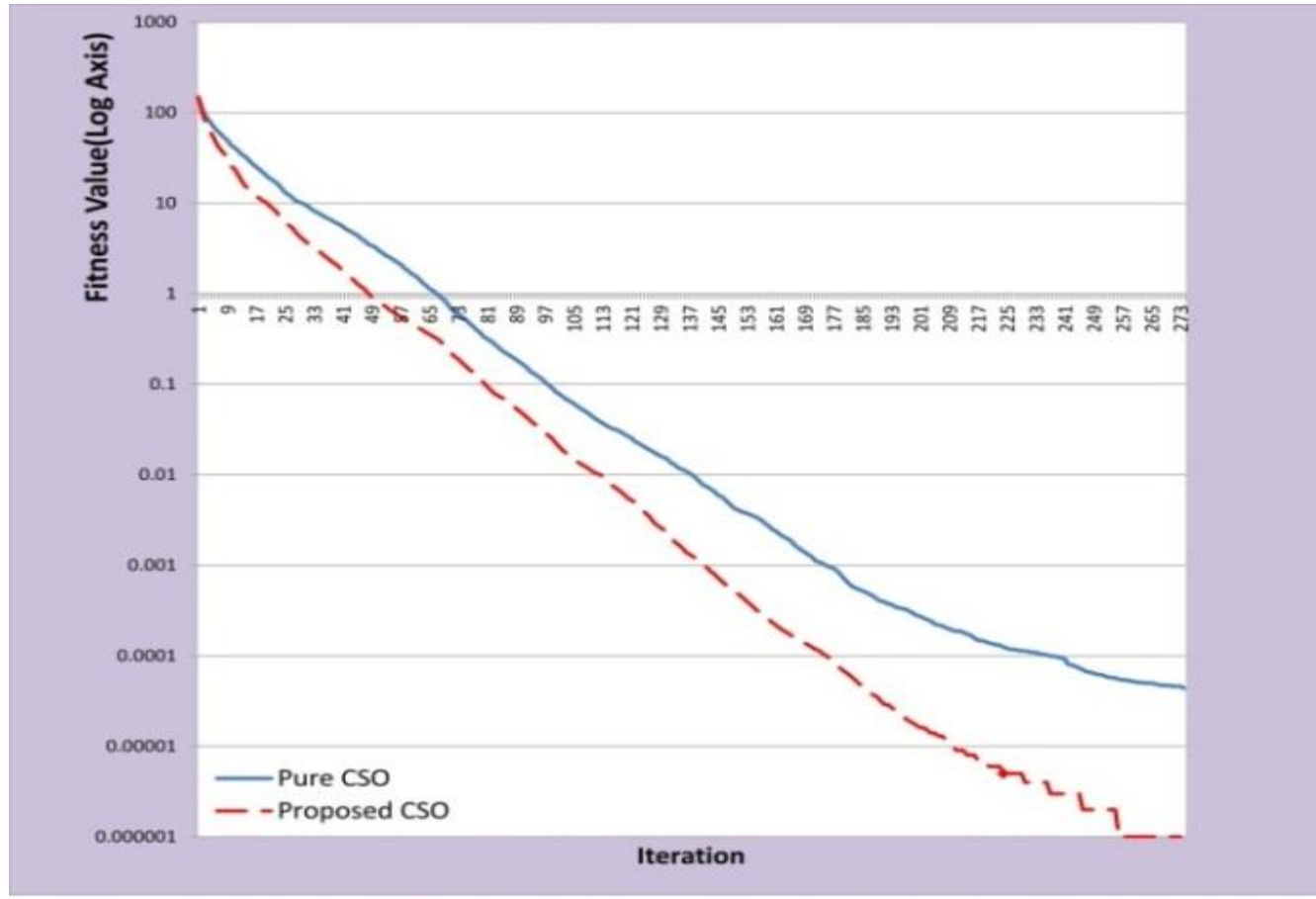

Fig. 6: Experimental Results of Zakharov Function

Figure 6 illustrates that the final solution of proposed CSO is better than pure CSO. It shows that proposed CSO can find the best answer in the 275th iteration which is 200 iterations less than pure CSO.

Trid 10 function is the last benchmark for making a comparison between pure CSO and proposed CSO. It has no local minimum except the global one. Equation (13) shows the equation of Trid10 function.

$$
F(x)=\sum_{i=1}^{10}\left(x_{i}-1\right)^{2}-\sum_{i=2}^{10} x_{i} x_{i-1}
$$

Table 9 and Figure 7 show that the best solution of proposed algorithm is equal to -194.000593 . In comparison with pure CSO, this value is closer to global minimum of function. (It is necessary to say that, due to many negative fitness values of Trid10 function, we added up the fitness values with number +194 in order to show the picture in log axis in Figure 7.

\section{Conclusion}

In the optimization theory, many algorithms are proposed and some of them are based on swarm intelligence. These types of algorithms imitate the behavior of animals. Cat Swarm Optimization (CSO) is a new optimization algorith $\mathrm{m}$ for finding the best global solution of a function which imitates the behavior of cats and models into two modes. In comparis on with Particle Swarm Optimization CSO can find the better global solution. But CSO is more complex than PSO and because of its complexity, sometimes takes a long time to find the best solution. So, by improving the pure $\mathrm{CSO}$, we proposed a new algorithm namely 'Adaptive Dynamic Cat Swarm Optimization'. First of all, we added an adaptive inertia weight to velocity update equation in order to facilitate the global and local search ability adaptively. Also we used an adaptive formula for updating the acceleration coefficient. Then, we used a new form of position update equation composing the novel position and velocity information. In new position equation, in addition to current information of position and velocity, we used the average information of two previous/next steps by applying a forgetting factor. Experimental results of six benchmarks indicated that the improved CSO in comparison with pure CSO has much better solution and achieves the best fitness value and converge in less iteration. Also by considering the process time, we found that our proposed algorithm runs faster than pure CSO.

\section{References}

[1] Dorigo, M. 1997, Ant colony system: a cooperative learning approach to the traveling salesman problem, IEEE Trans. on Evolutionary Computation. 26 (1) pp 53-66.

[2] Eberhart, R, Kennedy, J. 1995, A new optimizer using particle swarm theory, Sixth International Symposium on Micro Machine and Human Science,pp 39-43.

[3] Karaboga, D. 2005, An idea based on Honey Bee swarm for numerical optimisation, Technical 
Report TR06, Erciyes University, Engineering Faculty, Computer Engineering Department.

[4] Chu, S.C, Tsai, P.W, and Pan, J.S. 2006, Cat Swarm Optimization, LNAI 4099, 3 (1), Berlin Heidelberg: Springer-Verlag, pp. 854- 858.

[5] Santosa, B, and Ningrum,M. 2009, Cat Swarm Optimization for Clustering, International Conference of Soft Computing and Pattern Recognition, pp 54-59.

[6] Chu, S.C, Roddick, J.F, and Pan, J.S. 2004, Ant colony system with communication strategies, Information Sciences 167,pp 63-76

[7] Shi, Y, and Eberhart, R. 1999, Empirical study of particle swarm optimization, Congress on Evolutionary Computation,pp 1945-1950.

[8] Molga,M, and Smutnicki,C. 2005, Test functions for optimization needs", 3 kwietnia.

[9] Orouskhani,M, Mansouri, $M$ and Teshnehlab,M. 2011, Average-Inertia weighted Cat swarm optimization, LNCS, Berlin Heidelberg: SpringerVerlag, pp 321- 328.

\section{Authors' Profiles}

Meysam Orouskhani: $\mathrm{PhD}$ student of computer engineering in Science and Research branch of Islamic Azad University, major in Evolutionary Computation and Optimization.

Yasin Orouskhani: BSc student of computer engineering in Sharif University of Technology, major in Programming and foundation of Operating Systems.

Mohammad Mansouri: PhD student of Electrical and Computer engineering department in K.N.Toosi University, major in intelligent control.

Mohammad Teshnehlab: Professor of Control Engineering, Industrial Control Center of Excellence, Faculty of Electrical and Computer Engineering in K.N.Toosi University of Technology.

How to cite this paper: Meysam Orouskhani, Yasin Orouskhani, Mohammad Mansouri, Mohammad Teshnehlab,"A Novel Cat Swarm Optimization Algorithm for Unconstrained Optimization Problems", International Journal of Information Technology and Computer Science(IJITCS), vol.5, no.11, pp.32-41, 2013. DOI: 10.5815/ijitcs.2013.11.04 